OPEN ACCESS

Edited by:

Giorgio Malpeli,

University of Verona, Italy

Reviewed by:

Ormond MacDougald,

University of Michigan, United States

Stuart Rushworth,

University of East Anglia,

United Kingdom

${ }^{*}$ Correspondence:

Sicheng Wang

w.s.c@sina.com

Xiao Chen

sirchenxiao@126.com

Jiacan Su

drsujiacan@163.com

${ }^{t}$ These authors have contributed equally to this work

Specialty section:

This article was submitted to

Cellular Biochemistry,

a section of the journal

Frontiers in Cell and Developmental

Biology

Received: 04 September 2021

Accepted: 29 October 2021

Published: 29 November 2021

Citation:

Wang L, Zhang $H$, Wang S, Chen X and Su J (2021) Bone Marrow

Adipocytes: A Critical Player in the

Bone Marrow Microenvironment.

Front. Cell Dev. Biol. 9:770705.

doi: $10.3389 /$ fcell.2021.770705

\section{Bone Marrow Adipocytes: A Critical Player in the Bone Marrow Microenvironment}

\author{
Lipeng Wang ${ }^{1 \dagger}$, Hao Zhang ${ }^{2 \dagger}$, Sicheng Wang ${ }^{3 *}$, Xiao $\mathrm{Chen}^{2 *}$ and Jiacan $\mathrm{Su}^{1,2 *}$ \\ ${ }^{1}$ Institute of Translational Medicine, Shanghai University, Shanghai, China, ${ }^{2}$ Department of Orthopedics Trauma, Shanghai \\ Changhai Hospital, Naval Medical University, Shanghai, China, ${ }^{3}$ Department of Orthopedics, Shanghai Zhongye Hospital, \\ Shanghai, China
}

Recognized for nearly 100 years, bone marrow adipocytes (BMAs) form bone marrow niches that contain hematopoietic and bone cells, the roles of which have long been underestimated. Distinct from canonical white, brown, and beige adipocytes, BMAs derived from bone marrow mesenchymal stromal cells possess unique characteristics and functions. Recent single-cell sequencing studies have revealed the differentiation pathway, and seminal works support the tenet that BMAs are critical regulators in hematopoiesis, osteogenesis, and osteoclastogenesis. In this review, we discuss the origin and differentiation of BMAs, as well as the roles of BMAs in hematopoiesis, osteogenesis, osteoclastogenesis, and immune regulation. Overall, BMAs represent a novel target for bone marrow-related diseases, including osteoporosis and leukemia.

\begin{abstract}
Keywords: bone marrow adipocytes, bone marrow mesenchymal stromal cells, hematopoiesis, osteogenesis, osteoclastogenesis, immune regulation
\end{abstract}

\section{INTRODUCTION}

Bone marrow niches are specialized microenvironments that include hematopoietic cells, mesenchymal lineage cells, endothelial cells, and nerves. Erythroid, myeloid, and lymphoid cells constitute the hematopoietic lineage, and mesenchymal stromal cells (MSCs) mainly differentiate into adipocytes, osteoblasts, and chondrocytes (de Paula and Rosen, 2020).

Bone marrow adipocytes (BMAs) were first described in 1922, but it was not until recently that scientists began to understand the basic tenets of BMAs. BMAs are developmentally and functionally distinct from classical white, brown, and beige adipocytes (Sebo et al., 2019). White adipocytes are large, lipid-laden cells that act as an energy source for other tissues and make up $99 \%$ of the volume of the subcutaneous and visceral adipose tissue depot. Brown adipocytes are small, mitochondria-abundant cells that burn fatty acids to generate heat. Beige adipocytes reside in subcutaneous depots together with white adipocytes but share some functional features of brown adipocytes, especially in terms of thermogenic capacity through uncoupling protein 1 and morphology (multilocular small cells). BMAs, derived from a unique origin, are metabolically active cells with abundant lipid stores, mitochondria, and endoplasmic reticulum. The amount of BMAs is dynamic during development and in several conditions including osteoporosis, aging, and caloric restriction (de Paula and Rosen, 2020). These observations have encouraged the scientists to explore the roles of BMAs. 


\section{BMA IDENTIFICATION}

\section{Origin and classification}

Lineage tracing results have indicated that BMAs arise from bone marrow mesenchymal stromal cells (BMSCs), not white adipocytes, brown adipocytes, or hematopoietic progenitors. Efforts are ongoing to identify the adipocyte progenitors in vivo. It remains unclear whether BMAs originate from a single population or different sources.

Lepr $^{+}$BMSCs are a major source of BMAs in adults. BMSCs are heterogeneous and labeled according to the absence of hematopoietic or endothelial markers accompanied by the expression of several stem cell markers (Zhou et al., 2014). In adults, Lepr $^{+}$stromal cells are situated around the vascular network and account for $94 \%$ of BMSCs. Lepr $^{+}$cells arise postnatally and form most bone cells and BMAs in adults (Zhou et al., 2014). After irradiation, fracture, or transplantation, $\mathrm{Lepr}^{+}$cells are accountable for the generation of bone and BMAs (Zhou et al., 2014).

Comprising approximately $70 \%$ of adult marrow volume and $10 \%$ of adipose tissue mass in healthy individuals, bone marrow adipose tissue (BMAT) is mainly formed by BMAs and two distinct compositions exist: constitutive (cBMAT) and regulated (rBMAT) compartments (Scheller et al., 2015). Micro-computed tomography (CT) on osmium tetroxidestained bone imaging has shown that cBMAT is located in the distal region of the tibia. cBMAT forms immediately after birth and represents a more stable form of BMAT that rarely responds to environmental stimuli. cBMAT development results in an accumulation of cells. rBMAT resides in vertebrae and the proximal region of the tibia and is sensitive to global and local cues, including cold exposure and radiation; it also participates in physiological adaptation. Although the classification of rBMAT and cBMAT is clear within rodents, the extent to which this nomenclature can be applied to humans remains to be determined.

\section{Differentiation}

Although the differentiation routes of BMSCs into adipocytes have been elucidated, the identification and characteristics of the precursors are poorly explored. A recent study depicts the adipose precursor hierarchy of BMSCs (Ambrosi et al., 2017). Focusing on cell surface markers, they showed that $\mathrm{CD} 45^{-} \mathrm{CD} 31^{-} \mathrm{Sca}^{+} \mathrm{CD} 24^{+}$multipotent precursor cells differentiate into both BMAs and osteoblasts. CD45 and CD31 are hematopoietic lineage markers, while Sca and CD24 are adipose precursor markers. This population further differentiates into $\mathrm{CD} 45^{-} \mathrm{CD} 31^{-} \mathrm{Sca}^{+} \mathrm{CD} 24^{-}$adipogenic precursors, which give rise to $\mathrm{CD} 45^{-} \mathrm{CD} 31^{-} \mathrm{Sca}{ }^{-} \mathrm{Zfp} 234^{+}$ preadipocytes and then mature BMAs (Grandl and Wolfrum, 2017). Another group identifies new subtypes of Lepr $^{+}$BMSCs that differentiate into BMAs: $M p g^{\text {high }}$ and $L p l^{h^{\text {high }}}$ clusters (Tikhonova et al., 2019).

To delineate the development of BMSCs into terminal BMAs through hierarchical differentiation paths in vivo, Qin et al. performed extensive single-cell RNA-sequencing on BMSCs
(Zhong et al., 2020). Mesenchymal lineage cells are divided into nine subpopulations. Analysis of lineage-unique gene markers identified collections of BMAs, osteoblasts, osteocytes, and chondrocytes. Trajectory pattern analysis using the slingshot method identified the most primordial subgroup in the sequencing dataset: early mesenchymal progenitors (EMPs). The results show that EMPs express some stem cell markers, including Sca-1, Thy1, and Cd34. Based on the expression level of osteogenic genes, they also identified intermediate mesenchymal progenitors (IMPs), late mesenchymal progenitors (LMPs), and lineage committed progenitors (LCPs) before differentiation into osteoblasts or BMAs. The authors found that $\alpha$-smooth muscle actin (SMA) labels mesenchymal progenitors before bifurcated differentiation and can act as a marker for LMPs. Notably, they identified an original adipogenic lineage cell subpopulation, which are lipid-poor, labeled by adiponectin (Adipoq)-Cre and can be observed after LCPs and before mature BMAs during adipogenic differentiation. This novel population of adipogenic lineage cells is referred to as marrow adipogenic lineage precursors (MALPs). MALPs maintain marrow vasculature and inhibit bone formation by secreted factors such as vascular endothelial growth factor (VEGF) and angiopoietin 4 (ANGPT4) (Zhong et al., 2020). This study showed relatively comprehensive in vivo differentiation of BMSCs into BMAs inside the bone marrow (Figure 1). Overall, there is heterogeneity in adipocytes in bone marrow, and BMAs at different stages of differentiation may play distinct biological roles.

\section{ROLES OF BMAS}

Adipose tissue is a substantial endocrine organ that regulates physiological activities by secreting hormones and cytokines (Cawthorn et al., 2014; Suchacki et al., 2020). It is predicted that 263 distinct proteins are secreted by primary human adipocytes (Lehr et al., 2012). Although the function of BMAs in marrow niches is under investigation, their regulatory role in hematopoiesis, bone metabolism, and immunity are only partially understood.

\section{The role of BMAs in hematopoiesis is controversial}

Evidence indicates that BMAs negatively regulate hematopoiesis (Naveiras et al., 2009; Scheller et al., 2016). Adipocyte-abundant caudal vertebrae retain fewer hematopoietic stem cells (HSCs) and short-term progenitors compared with adipocyte-free thoracic vertebrae. Furthermore, the genetic and pharmacologic inhibition of adipogenesis accelerates hematopoietic recovery after irradiation and bone marrow transplant (Naveiras et al., 2009). However, it is still unclear if this reflects the influence of BMAs on HSCs or a secondary impact on the marrow environment.

A recent study reported that adipocytes in long bones support hematopoietic restoration following irradiation by providing stem cell factor (SCF), a critical factor for HSC survival 


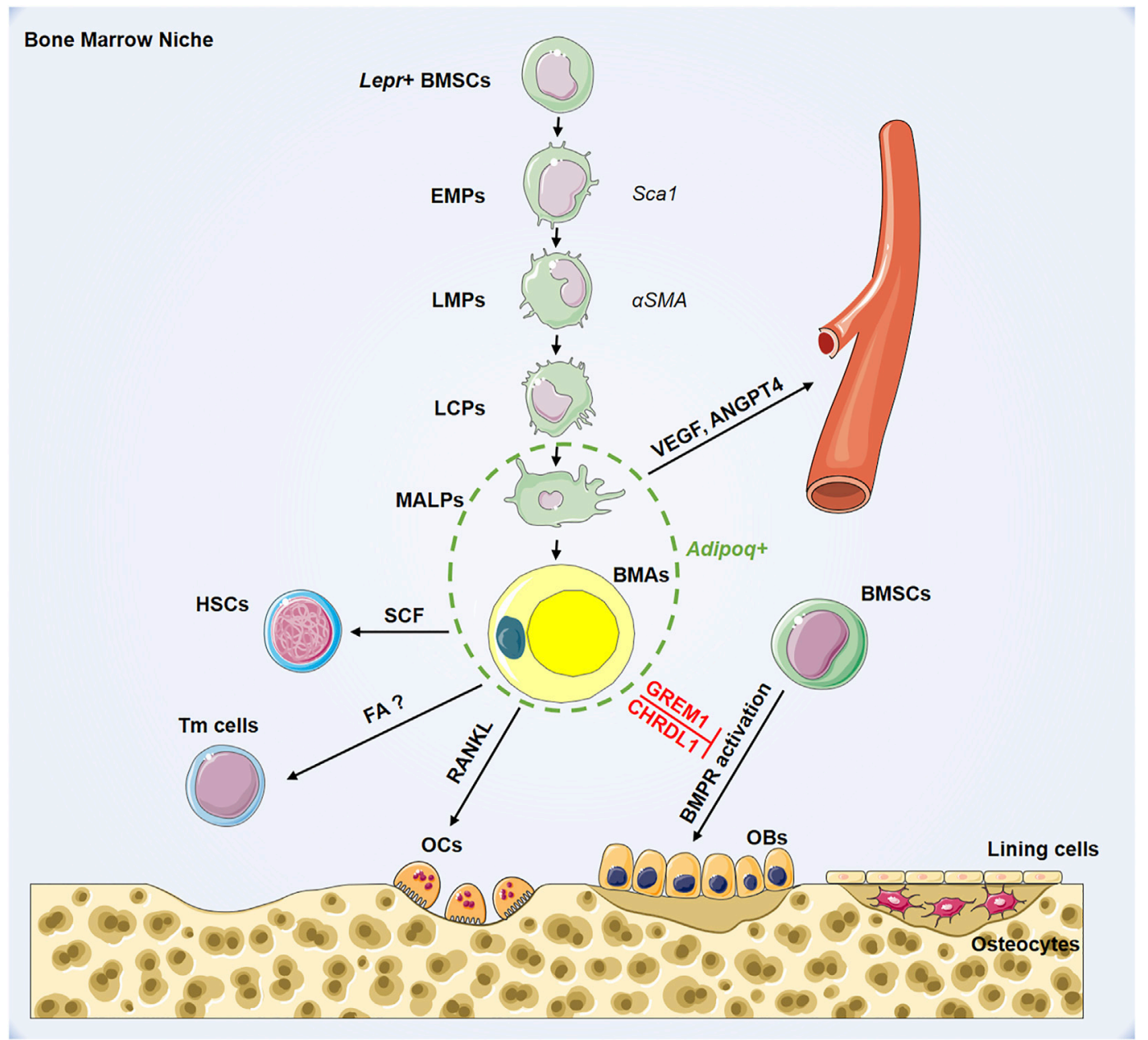

FIGURE 1 | Hierarchy of bone marrow adipocyte lineage cell and its role in bone marrow niche. BMSCs: bone marrow mesenchymal stromal cells; EMPs: early mesenchymal progenitors; LMPs: late mesenchymal progenitors; LCPs: lineage committed progenitors; MALPs: marrow adipogenic lineage precursors; BMAs: bone marrow adipocytes; HSCs: hematopoietic stem cells; Tmcells: T memory cells; OCs: osteoclasts; OBs: osteoblasts; Lepr: leptin receptor; Sca1: stem cell antigen 1;

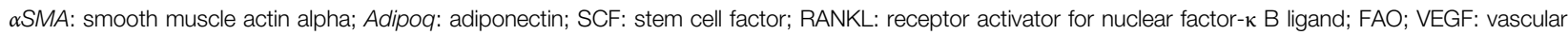
endothelial growth factor; ANGPT4: angiopoietin 4; GREM1: gremlin1; CHRDL1: chordin-like1; BMPR: bone morphogenetic protein receptor.

(Oguro et al., 2013), whereas in tail vertebrae adipocytes impair hematopoiesis (Zhou et al., 2017). After irradiation, fatless A-ZIP/F1 mice have reduced overall marrow cells and HSCs in long bones, yet exhibit an increased number in caudal vertebrae (Zhou et al., 2017). This difference was attributed to the presence of a high number of blood vessels in the tail vertebrae of A-ZIP/F1 mice, a phenomenon not seen in femurs (Zhou et al., 2017). Suppression of marrow vascularization impairs HSC frequency and hematopoietic regeneration (Hooper et al., 2009). In addition to SCF, BMAs also synthesize adiponectin and leptin, which promote HSC proliferation (DiMascio et al., 2007; Poloni et al., 2013). Although the finding that BMA expansion is accompanied by reduced hematopoiesis has conventionally been interpreted to reflect an inhibitory effect of BMAs on hematopoiesis, these data suggest that adipogenesis is an emergency response that produces HSC niche factors and promotes hematopoiesis in most bones. Compared with constructing new perivascular niches, adipogenesis is a faster way to produce HSC niche factors, which involves the promotion of marrow vascularization (Zhou et al., 2017).

To confirm further the connections between BMAs and hematopoiesis in primates, the rhesus macaque model has been used to determine that hematopoietic stem and progenitor cells (HSPCs) reside abreast of BMAs. Furthermore, BMAT-conditioned medium promotes the expansion and differentiation of HSPCs ex vivo (Robino et al., 2020). To explore the underlying mechanism, quantitative proteomic examination of BMAT-conditioned medium was performed. A total of 994 proteins were found to be released from BMAT, including TGFB1, FBLN1, IGFBP2, LGALS1, TIMP1, and C3, which have been identified as positive regulators of HSPC differentiation, motility, and adhesion (Robino et al., 2020). Among them, 430 proteins are of microvesicular/exosomal origin, indicating complex 
composition and paracrine activity. Of note, BMAT contains many types of cells in addition to BMAs, including granulocytes and monocytes/macrophages (Robino et al., 2020). Several proteins identified from BMAT are derived from these cellular neighborhoods of BMAs. Thus, BMAs may also regulate HSPC activity through these immune cells.

The function of BMAs in leukemia is debatable and lineage specific. In acute lymphoblastic leukemia (ALL), in vitro and in vivo studies indicate that BMAs inhibit T-ALL proliferation (Cahu et al., 2017). In acute myeloid leukemia (AML), Shafat et al. report that AML blasts cocultured with BMA show reduced apoptosis and enhanced proliferation (Shafat et al., 2017). AML blasts promote BMA lipolysis, and the fatty acids generated are transmitted from BMAs to AML cells for $\beta$-oxidation. However, a recent study showed that AML caused a reduction in adipocytes in human marrow and AML xenografts (Boyd et al., 2017), suggesting that AML mainly influences the adipocyte population, in addition to promoting the lipolysis of preexisting adipocytes (Boyd et al., 2017). Furthermore, global transcriptome analysis of BMSCs from AML patients or healthy bone marrow donors revealed that adipogenic differentiation is compromised by AML (Boyd et al., 2017). To explore further the relationship between BMA decline and deficient myeloerythropoiesis in AML, the researchers performed Transwell assays, which showed that BMAs promote myeloid and erythroid lineage maturation. The PPAR $\gamma$ agonist GW1929 was used to stimulate adipogenesis and was found to rescue hematopoietic maturation while suppressing leukemic growth (Boyd et al., 2017). Overall, BMAs promote normal myeloerythroid maturation and may be a useful therapeutic target to improve bone marrow failure in AML. However, the high sensitivity of BMAs to the change of metabolic status hampers a clear definition of its function in distinct clinical situations and further studies are needed to fully unveil the function of BMA in physiology and different pathology conditions (Zinngrebe et al., 2020).

\section{BMAs inhibit osteogenesis}

Findings in healthy individuals indicate that regardless of age (age 5-88 years), BMAs are negatively related to bone mass (Shen et al., 2007; Shen et al., 2014). BMAs have also been reported to inhibit bone formation and fracture healing, although the underlying mechanism is still under investigation (Ambrosi et al., 2017).

BMA ablation enhances osteogenesis. In a recent study, researchers mated mice bearing diphtheria toxin receptor (DTR), under control of a STOP-flox, to Adipoq-Cre mice (Zou et al., 2020). The DTR ${ }^{\text {Adipoq }}$ mice showed eliminated peripheral and marrow adipocytes following administration of the diphtheria toxin. The bone mass of Adipoq-deficiency mice increased 10-fold with 10 days of diphtheria toxin treatment. To exclude the effect of peripheral adipocytes, the authors performed parabiosis between wild-type and $\mathrm{DTR}^{\text {Adipoq }}$ mice and found that diphtheria toxin treatment to control mice induced $\mathrm{DTR}^{\text {Adipoq }}$ mice osteosclerosis, while administration of diphtheria toxin to $\mathrm{DTR}^{\text {Adipoq }}$ mice had no impact on the bone volume of wild-type partners (Zou et al., 2020). Thus, enhanced osteogenesis in
DTR $^{\text {Adipoq }}$ mice is mediated by BMA ablation. To uncover the mechanism of enhanced bone formation following BMA deletion, they further mated $\mathrm{DTR}^{\text {Adipoq }}$ mice to 2.3Col-GFP reporter mice to characterize osteoblasts. Four days after diphtheria toxin induction, more $\mathrm{GFP}^{+}$cells were observed in $\mathrm{DTR}^{\text {Adipoq }}$ mice. They next mated $\mathrm{DTR}^{\text {Adipoq }} 2.3 \mathrm{Col}$-GFP mice to $T K-3.6$ Col1a1 mice, a specific strain transduced by mitotic preosteoblastic cells (Zou et al., 2020). Diphtheria toxin-induced osteosclerosis is reversed in TK-3.6Col1a1 mice by ganciclovir, an agent that targets replicating pre-osteoblasts (Zou et al., 2020). These results suggest that BMA ablation promotes pre-osteoblast recruitment and their differentiation into mature osteoblasts. This impact is a result of activation of bone morphogenetic protein receptor (BMPR) and epidermal growth factor receptor pathways. BMAs express chordin-like1 (CHRDL1) and gremlin1 (GREM1), specific BMPR inhibitors, and thus suppress osteogenesis (Zou et al., 2020). Another group reported that BMAs also secrete interleukin (IL)-6 and palmitate to suppress osteoblast activity (Gasparrini et al., 2009; Gunaratnam et al., 2014).

BMAs mediate myeloma-induced osteoblastogenesis suppression. Multiple myeloma is distinguished by overactive bone absorption and impaired bone generation (Palumbo and Anderson, 2011). When BMSCs are cultured with conditioned medium from BMAs obtained from myeloma patients or preexposed to myeloma cells, the researchers observed reduced Alizarin red S staining, alkaline phosphatase levels, and osteoblastic gene expression (Liu et al., 2019). Furthermore, using an extramedullary model of osteogenesis and calvarial bone defect, they found that myeloma-associated adipocytes weakened new bone formation (Liu et al., 2019). To explore the mechanism, microarray analysis and $\mathrm{qPCR}$ were performed to examine adipokine expression profiles in adipocytes from the bone marrow of patients and healthy controls. Three downregulated genes (adiponectin, adipsin, and visfatin) and one upregulated gene (Tnfa) were identified. The changes in expression of these adipokines inhibited osteoblastogenesis (Liu et al., 2019).

BMAs and osteoblasts are derived from the same stem cell, and the direction toward adipocytes occurs at the cost of osteoblast reduction. Thus, regulating the lineage allocation of BMSCs is an effective way to enhance osteoblastogenesis (Suresh et al., 2019; Yu et al., 2019). However, it is notable that the mutual exclusivity between adipocytes and osteoblasts in BM has not yet been proven. During puberty, bone marrow changes from red toward yellow but bone-forming activity has reached its peak (Moore and Dawson, 1990; Devlin and Rosen, 2015). Various animal models show elevated bone mass and excessive BMAT (Ackert-Bicknell et al., 2009).

\section{BMAs enhance osteoclastogenesis}

BMAs promote osteoclastogenesis and recent studies by $\mathrm{Hu}$ et al. and $\mathrm{Yu}$ et al. have begun to address how BMAs regulate osteoclast formation and bone remodeling (Hu et al., 2021b; Yu et al., 2021). Receptor activator of NF- $\kappa B$ (RANK) and its ligand RANKL play pivotal roles in osteoclastogenesis (Theill et al., 2002). RANKL is predominantly found in osteoblasts, osteocytes, and hypertrophic 
chondrocytes and binds RANK of osteoclast progenitors to induce osteoclastogenesis (Onji et al., 2021). Notably, BMAs also express RANKL (Fan et al., 2017). Yu et al. crossed Rankl-floxed mice with the Adipoq-Cre line to uniquely knock out Rankl in adipogenic lineage cells $\left(\right.$ Adipoq $^{\mathrm{cre}} ; \operatorname{Rankl}^{\mathrm{fl} / \mathrm{fl}}$ ). Under physiological conditions, these mice showed impaired osteoclastogenesis and bone resorption (Yu et al., 2021). In ovariectomy-induced osteoporosis, we found no decrease in cancellous bone density or cortical bone thickness after Rankl deletion in adipose cells (Hu et al., 2021b). To explore the role of RANKL in pathological BMA expansion, Adipo $^{\text {cre }} ; R_{a n k} l^{\mathrm{fl} / \mathrm{fl}}$ mice were administered the PPAR $\gamma$ activator rosiglitazone to increase bone marrow adipogenesis and the possibility of fracture (Aubert et al., 2010). Adipoq ${ }^{\text {cre }} ;$ Rankl $^{\mathrm{fl} / \mathrm{fl}}$ mice showed similar BMA expansion but a reduced number of osteoclasts compared with control Rankl ${ }^{\mathrm{fl} / \mathrm{fl}}$ mice (Aubert et al., 2010). These findings suggest that expanded BMA are crucial sources of RANKL for increased osteoclastogenesis and bone resorption. Overall, these two independent studies revealed that BMAs mediate bone remodeling through RANK/RANKL-dependent regulation of osteoclastogenesis in physiological and pathological states. Thus, targeting bone marrow adipogenesis and RANKL signaling in BMAs may be useful to treat osteoporosis.

\section{BMAs regulate immune function}

BMAs contribute to inflammation and plasma cell malfunction inside the bone marrow. Memory T cells and long-lasting plasma cells settle primarily in the marrow to provide protection against recurrent infections (Tokoyoda et al., 2009). To investigate the function of BMAs in immune regulation, global gene expression analysis was performed to compare mRNA expression of adipocytes from human BMAs and white adipose tissue (WAT) (Miggitsch et al., 2019). Several cytokines, including CCL2 CCL5, IL6, IL8, IL10, IL15, CCR7, CCRL2, and CXCL1 were elevated in BMAs, indicating its immune regulatory function within the bone marrow (Miggitsch et al., 2019). Furthermore, the production of reactive oxygen species (ROS) is elevated inside BMAs. ROS secretion accounts for the inhibition of IgG producing plasma cells (Miggitsch et al., 2019).

BMAs promote memory $\mathrm{T}$ cell gathering in the bone marrow upon dietary restriction (DR). While WAT collapses after caloric restriction, BMAs are paradoxically increased, with unclear significance. Recent findings have revealed that memory $\mathrm{T}$ cells redistribute from the periphery toward the bone marrow in response to the nutritional challenge (Collins et al., 2019). To determine if BMA expansion contributes to memory $\mathrm{T}$ cell viability and aggregation, the Adipoq-Cre ${ }^{\mathrm{ERT} 2} \times$ Rosa26DTA mice were generated to delete BMAs. Rosa26-DTA mice carry a loxP-flanked stop cassette linked to the active fragment of diphtheria toxin. When crossed with Adipoq-Cre mice, specific ablation of adipocytes is achieved. In mice with reduced BMAs, memory $\mathrm{T}$ cells are no longer sustained in the marrow after DR, indicating BMAs are important for memory $\mathrm{T}$ cells homing to the bone marrow. Moreover, these $\mathrm{T}$ cells show strengthened protection resisting secondary cancers and infections (Collins et al., 2019). Previous studies have revealed that long-chain fatty acids are indispensable for $\mathrm{T}$ cell survival, but how BMAs contribute to memory $\mathrm{T}$ cell maintenance and homing is still unclear. Collectively, BMAs help maintain and optimize immunological retention upon DR with an unknown mechanism.

\section{REGULATION OF BONE MARROW ADIPOGENESIS}

An increase in BMAT is a shared reaction to various clinical circumstances and medication, such as diabetes, obesity, anorexia, senescence, and glucocorticoid treatment. Based on the role of BMAs, regulating bone marrow adipogenesis is a promising method for treating bone marrow-related diseases and controlling the differentiation fate of BMSCs is a feasible way.

Several regulators participate in BMSC differentiation. $\operatorname{PPAR} \gamma, \mathrm{C} / \mathrm{EBP} \alpha$, platelet-derived growth factor receptor $\beta$ and zinc finger proteins 423, 467, and 521 are well-known factors required for adipogenesis (de Paula and Rosen, 2020); additional regulators are under investigation. Forkhead box P1 (FOXP1) can interact with the CEBP $\beta / \delta$ complex and RBPjк to regulate BMSC fate switches (Li et al., 2017). Expressed on BMSCs, Thy-1 (CD90) is a glycosylphosphatidyl-anchored protein of the immunoglobulin family. Thy-1-deficient mice show increased adipogenesis (Picke et al., 2018), and Thy-1 deficiency results in a reduction in Wnt ligand concomitantly with upregulation of the Wnt inhibitors dickkopt-1 and sclerostin, which inhibit osteogenesis (Picke et al., 2018). microRNAs (miRs) participate in cell metabolism by regulating the mRNA degradation of target mRNA. Antagonism of miR-188 can affect the differentiation fate of BMSCs and promote bone formation ( $\mathrm{Hu}$ et al., 2021a). BMSC differentiation is also epigenetically regulated: histone demethylases KDM4B and KDM6B inhibit adipogenic differentiation of BMSCs via elimination of H3K9me3 and H3K27me3 (Ye et al., 2012).

The bone marrow niche also provides information that regulates BMSC lineage commitment. Sensory nerves can induce osteogenic differentiation of BMSCs by downregulating sympathetic nerve activity. Local elevation of prostaglandin E2 triggers EP4 receptors in sensory nerves and inhibits adipogenesis (Hu et al., 2020). Mechanical forces facilitate osteogenic differentiation of BMSCs and prohibit BMA production (Ozcivici et al., 2010). During mechanical loading, modulation of actin regulates ERK and AKT pathways to induce BMSC differentiation ( $\mathrm{Li}$ et al., 2015). mTORC2 also plays a role in strain-induced cytoskeletal reorganization. Deletion of mTORC2 in BMSCs abolishes osteogenic differentiation and facilitates adipogenic differentiation (Sen et al., 2014). Endocrine molecules also influence BMSC differentiation. Estrogen acts on estrogen receptor- $\alpha$ and has been shown to suppress adipogenesis (Rooney and van der Meulen, 2017). Both BMSCs and BMAs express the follicle-stimulating hormone (FSH) receptor, and inhibition of its interaction with $\mathrm{FSH}$ prevents adipogenesis (Liu et al., 2017). Parathyroid hormone (PTH) regulates bone metabolism and inhibits the differentiation of BMSCs toward BMAs (Fan et al., 2017). Moreover, BMAs express PTH1R, and PTH can induce adipogenic lipolysis, which further diminishes adipogenesis in the bone marrow niche 
(Maridas et al., 2019). Leptin also regulates bone metabolism (Cohen et al., 1996); hypothalamic or subcutaneous administration of leptin has been shown to impair obesityinduced marrow adiposity (Hamrick et al., 2005; Ambati et al., 2010). Overall, several factors control BMSCs differentiation fate, and further work are needed to identify novel regulators.

\section{PERSPECTIVE}

BMAs are unique adipocytes that reside in the skeletal space. Previous studies have suggested that mature and premature adipocytes exert various influences on hematopoiesis, bone remodeling, and immune regulation in bone marrow niches (Figure 1). However, it is worth noting that the specificity of Adipoq-Cre is questionable (Onji et al., 2021). Many studies have targeted BMAs with Adipoq-Cre; however, in aged mice, some osteocytes and osteoblasts are also Crepositive (Mukohira et al., 2019; Yu et al., 2021). Moreover, Adipoq is a marker of mature adipocytes as well as their progenitors, MALPs (Yu et al., 2021). Further studies using more specific Cre lines are warranted to uncover the function of BMAs and adipocyte precursors in distinct phases of differentiation.

\section{REFERENCES}

Ackert-Bicknell, C. L., Shockley, K. R., Horton, L. G., Lecka-Czernik, B., Churchill, G. A., and Rosen, C. J. (2009). Strain-specific Effects of Rosiglitazone on Bone Mass, Body Composition, and Serum Insulin-like Growth Factor-I. Endocrinology 150 (3), 1330-1340. doi:10.1210/en.2008-0936

Ambati, S., Li, Q., Rayalam, S., Hartzell, D. L., Della-Fera, M. A., Hamrick, M. W., et al. (2010). Central Leptin versus Ghrelin: Effects on Bone Marrow Adiposity and Gene Expression. Endocr 37 (1), 115-123. doi:10.1007/s12020-009-9274-Z

Ambrosi, T. H., Scialdone, A., Graja, A., Gohlke, S., Jank, A.-M., Bocian, C., et al. (2017). Adipocyte Accumulation in the Bone Marrow during Obesity and Aging Impairs Stem Cell-Based Hematopoietic and Bone Regeneration. Cell Stem Cell 20 (6), 771-784. e776. doi:10.1016/ j.stem.2017.02.009

Aubert, R. E., Herrera, V., Chen, W., Haffner, S. M., and Pendergrass, M. (2010). Rosiglitazone and Pioglitazone Increase Fracture Risk in Women and Men with Type 2 Diabetes. Diabetes Obes. Metab. 12 (8), 716-721. doi:10.1111/j.14631326.2010.01225.x

Boyd, A. L., Reid, J. C., Salci, K. R., Aslostovar, L., Benoit, Y. D., Shapovalova, Z., et al. (2017). Acute Myeloid Leukaemia Disrupts Endogenous MyeloErythropoiesis by Compromising the Adipocyte Bone Marrow Niche. Nat. Cel Biol 19 (11), 1336-1347. doi:10.1038/ncb3625

Cahu, X., Calvo, J., Poglio, S., Prade, N., Colsch, B., Arcangeli, M.-L., et al. (2017). Bone Marrow Sites Differently Imprint Dormancy and Chemoresistance to T-Cell Acute Lymphoblastic Leukemia. Blood Adv. 1 (20), 1760-1772. doi:10.1182/bloodadvances.2017004960

Cawthorn, W. P., Scheller, E. L., Learman, B. S., Parlee, S. D., Simon, B. R., Mori, H., et al. (2014). Bone Marrow Adipose Tissue Is an Endocrine Organ that Contributes to Increased Circulating Adiponectin during Caloric Restriction. Cel Metab. 20 (2), 368-375. doi:10.1016/j.cmet.2014.06.003

Cohen, S. L., Halaas, J. L., Friedman, J. M., Chait, B. T., Bennett, L., Chang, D., et al. (1996). Human Leptin Characterization. Nature 382 (6592), 589. doi:10.1038/ 382589a0

Collins, N., Han, S.-J., Enamorado, M., Link, V. M., Huang, B., Moseman, E. A., et al. (2019). The Bone Marrow Protects and Optimizes Immunological
Lineage allocation of BMSCs is regulated by niche inputs that involve mechanical, neural, and endocrine modulators. Efforts are being made to genetically or pharmacologically manipulate bone marrow adipogenesis, and it may constitute a novel therapeutic strategy for bone marrow-related disorders.

\section{AUTHOR CONTRIBUTIONS}

LW, SW, XC, and JS conceived the article. LW and HZ wrote the article. SW, XC, and JC reviewed and edited the article. All authors listed have made a substantial, direct, and intellectual contribution to work and approved it for publication.

\section{FUNDING}

This project was supported by the National Key Research and Development Plan (2018YFC2001500), National Natural Science Foundation of China (NSFC) Key Research Program in Aging (91749204), National Natural Science Foundation of China (81771491, 81871099, 81972254, and 82172098), and Shanghai Rising-Star Program (21QA1412000).

Memory during Dietary Restriction. Cell 178 (5), 1088-1101. e1015. doi:10.1016/j.cell.2019.07.049

de Paula, F. J. A., and Rosen, C. J. (2020). Marrow Adipocytes: Origin, Structure, and Function. Annu. Rev. Physiol. 82, 461-484. doi:10.1146/annurev-physiol021119-034513

Devlin, M. J., and Rosen, C. J. (2015). The Bone-Fat Interface: Basic and Clinical Implications of Marrow Adiposity. Lancet Diabetes Endocrinol. 3 (2), 141-147. doi:10.1016/s2213-8587(14)70007-5

DiMascio, L., Voermans, C., Uqoezwa, M., Duncan, A., Lu, D., Wu, J., et al. (2007) Identification of Adiponectin as a Novel Hemopoietic Stem Cell Growth Factor. J. Immunol. 178 (6), 3511-3520. doi:10.4049/jimmunol.178.6.3511

Fan, Y., Hanai, J.-i., Le, P. T., Bi, R., Maridas, D., DeMambro, V., et al. (2017). Parathyroid Hormone Directs Bone Marrow Mesenchymal Cell Fate. Cel Metab. 25 (3), 661-672. doi:10.1016/j.cmet.2017.01.001

Gasparrini, M., Rivas, D., Elbaz, A., and Duque, G. (2009). Differential Expression of Cytokines in Subcutaneous and Marrow Fat of Aging C57BL/6J Mice. Exp. Gerontol. 44 (9), 613-618. doi:10.1016/j.exger.2009.05.009

Grandl, G., and Wolfrum, C. (2017). Adipocytes at the Core of Bone Function. Cell Stem Cell 20 (6), 739-740. doi:10.1016/j.stem.2017.05.008

Gunaratnam, K., Vidal, C., Gimble, J. M., and Duque, G. (2014). Mechanisms of Palmitate-Induced Lipotoxicity in Human Osteoblasts. Endocrinology 155 (1), 108-116. doi:10.1210/en.2013-1712

Hamrick, M. W., Della-Fera, M. A., Choi, Y.-H., Pennington, C., Hartzell, D., and Baile, C. A. (2005). Leptin Treatment Induces Loss of Bone Marrow Adipocytes and Increases Bone Formation in Leptin-Deficient Ob/ob Mice. J. Bone Miner Res. 20 (6), 994-1001. doi:10.1359/jbmr.050103

Hooper, A. T., Butler, J. M., Nolan, D. J., Kranz, A., Iida, K., Kobayashi, M., et al. (2009). Engraftment and Reconstitution of Hematopoiesis Is Dependent on VEGFR2-Mediated Regeneration of Sinusoidal Endothelial Cells. Cell Stem Cell 4 (3), 263-274. doi:10.1016/j.stem.2009.01.006

Hu, B., Lv, X., Chen, H., Xue, P., Gao, B., Wang, X., et al. (2020). Sensory Nerves Regulate Mesenchymal Stromal Cell Lineage Commitment by Tuning Sympathetic Tones. J. Clin. Invest. 130 (7), 3483-3498. doi:10.1172/jci131554

Hu, Y., Li, X., Zhang, Q., Gu, Z., Luo, Y., Guo, J., et al. (2021a). Exosomeguided Bone Targeted Delivery of Antagomir-188 as an Anabolic Therapy for Bone Loss. Bioactive Mater. 6 (9), 2905-2913. doi:10.1016/ j.bioactmat.2021.02.014 
Hu, Y., Li, X., Zhi, X., Cong, W., Huang, B., Chen, H., et al. (2021b). RANKL from Bone Marrow Adipose Lineage Cells Promotes Osteoclast Formation and Bone Loss. EMBO Rep. 22, e52481. doi:10.15252/embr.202152481

Lehr, S., Hartwig, S., Lamers, D., Famulla, S., Müller, S., Hanisch, F.-G., et al. (2012). Identification and Validation of Novel Adipokines Released from Primary Human Adipocytes. Mol. Cell Proteomics 11 (1), M111. doi:10.1074/mcp.M111.010504

Li, H., Liu, P., Xu, S., Li, Y., Dekker, J. D., Li, B., et al. (2017). FOXP1 Controls Mesenchymal Stem Cell Commitment and Senescence during Skeletal Aging. J. Clin. Invest. 127 (4), 1241-1253. doi:10.1172/jci89511

Li, R., Liang, L., Dou, Y., Huang, Z., Mo, H., Wang, Y., et al. (2015). Mechanical Strain Regulates Osteogenic and Adipogenic Differentiation of Bone Marrow Mesenchymal Stem Cells. Biomed. Res. Int. 2015, 1-10. doi:10.1155/2015/ 873251

Liu, H., He, J., Koh, S. P., Zhong, Y., Liu, Z., Wang, Z., et al. (2019). Reprogrammed Marrow Adipocytes Contribute to Myeloma-Induced Bone Disease. Sci. Transl. Med. 11 (494), eaau9087. doi:10.1126/scitranslmed.aau9087

Liu, P., Ji, Y., Yuen, T., Rendina-Ruedy, E., DeMambro, V. E., Dhawan, S., et al. (2017). Blocking FSH Induces Thermogenic Adipose Tissue and Reduces Body Fat. Nature 546 (7656), 107-112. doi:10.1038/nature22342

Maridas, D. E., Rendina-Ruedy, E., Helderman, R. C., DeMambro, V. E., Brooks, D., Guntur, A. R., et al. (2019). Progenitor Recruitment and Adipogenic Lipolysis Contribute to the Anabolic Actions of Parathyroid Hormone on the Skeleton. FASEB j. 33 (2), 2885-2898. doi:10.1096/ fj.201800948RR

Miggitsch, C., Meryk, A., Naismith, E., Pangrazzi, L., Ejaz, A., Jenewein, B., et al. (2019). Human Bone Marrow Adipocytes Display Distinct Immune Regulatory Properties. EBioMedicine 46, 387-398. doi:10.1016/ j.ebiom.2019.07.023

Moore, S. G., and Dawson, K. L. (1990). Red and Yellow Marrow in the Femur: Age-Related Changes in Appearance at MR Imaging. Radiology 175 (1), 219-223. doi:10.1148/radiology.175.1.2315484

Mukohira, H., Hara, T., Abe, S., Tani-Ichi, S., Sehara-Fujisawa, A., Nagasawa, T., et al. (2019). Mesenchymal Stromal Cells in Bone Marrow Express Adiponectin and Are Efficiently Targeted by an Adiponectin Promoter-Driven Cre Transgene. Int. Immunol. 31 (11), 729-742. doi:10.1093/intimm/dxz042

Naveiras, O., Nardi, V., Wenzel, P. L., Hauschka, P. V., Fahey, F., and Daley, G. Q. (2009). Bone-marrow Adipocytes as Negative Regulators of the Haematopoietic Microenvironment. Nature 460 (7252), 259-263. doi:10.1038/nature08099

Oguro, H., Ding, L., and Morrison, S. J. (2013). SLAM Family Markers Resolve Functionally Distinct Subpopulations of Hematopoietic Stem Cells and Multipotent Progenitors. Cell Stem Cell 13 (1), 102-116. doi:10.1016/ j.stem.2013.05.014

Onji, M., Werschler, N., and Penninger, J. (2021). A Critical Relationship between Bone and Fat: the Role of Bone Marrow Adipose-derived RANKL in Bone Metabolism. EMBO Rep. 22, e52986. doi:10.15252/embr.202152986

Ozcivici, E., Luu, Y. K., Adler, B., Qin, Y.-X., Rubin, J., Judex, S., et al. (2010). Mechanical Signals as Anabolic Agents in Bone. Nat. Rev. Rheumatol. 6 (1), 50-59. doi:10.1038/nrrheum.2009.239

Palumbo, A., and Anderson, K. (2011). Multiple Myeloma. N. Engl. J. Med. 364 (11), 1046-1060. doi:10.1056/NEJMra1011442

Picke, A.-K., Campbell, G. M., Blüher, M., Krügel, U., Schmidt, F. N., Tsourdi, E., et al. (2018). Thy-1 (CD90) Promotes Bone Formation and Protects against Obesity. Sci. Transl. Med. 10 (453), eaao6806. doi:10.1126/ scitranslmed.aao6806

Poloni, A., Maurizi, G., Serrani, F., Mancini, S., Zingaretti, M. C., Frontini, A., et al. (2013). Molecular and Functional Characterization of Human Bone Marrow Adipocytes. Exp. Hematol. 41 (6), 558-566. e552. doi:10.1016/ j.exphem.2013.02.005

Robino, J. J., Pamir, N., Rosario, S., Crawford, L. B., Burwitz, B. J., Roberts, C. T., Jr., et al. (2020). Spatial and Biochemical Interactions between Bone Marrow Adipose Tissue and Hematopoietic Stem and Progenitor Cells in Rhesus Macaques. Bone 133, 115248. doi:10.1016/j.bone.2020.115248

Rooney, A. M., and van der Meulen, M. C. H. (2017). Mouse Models to Evaluate the Role of Estrogen Receptor a in Skeletal Maintenance and Adaptation. Ann. N.Y. Acad. Sci. 1410 (1), 85-92. doi:10.1111/nyas.13523
Scheller, E. L., Cawthorn, W. P., Burr, A. A., Horowitz, M. C., and MacDougald, O. A. (2016). Marrow Adipose Tissue: Trimming the Fat. Trends Endocrinol. Metab. 27 (6), 392-403. doi:10.1016/j.tem.2016.03.016

Scheller, E. L., Doucette, C. R., Learman, B. S., Cawthorn, W. P., Khandaker, S., Schell, B., et al. (2015). Region-specific Variation in the Properties of Skeletal Adipocytes Reveals Regulated and Constitutive Marrow Adipose Tissues. Nat. Commun. 6, 7808. doi:10.1038/ncomms8808

Sebo, Z. L., Rendina-Ruedy, E., Ables, G. P., Lindskog, D. M., Rodeheffer, M. S., Fazeli, P. K., et al. (2019). Bone Marrow Adiposity: Basic and Clinical Implications. Endocr. Rev. 40 (5), 1187-1206. doi:10.1210/ er.2018-00138

Sen, B., Xie, Z., Case, N., Thompson, W. R., Uzer, G., Styner, M., et al. (2014). mTORC2 Regulates Mechanically Induced Cytoskeletal Reorganization and Lineage Selection in Marrow-Derived Mesenchymal Stem Cells. J. Bone Miner Res. 29 (1), 78-89. doi:10.1002/jbmr.2031

Shafat, M. S., Oellerich, T., Mohr, S., Robinson, S. D., Edwards, D. R., Marlein, C. R., et al. (2017). Leukemic Blasts Program Bone Marrow Adipocytes to Generate a Protumoral Microenvironment. Blood 129 (10), 1320-1332. doi:10.1182/blood-2016-08-734798

Shen, W., Chen, J., Punyanitya, M., Shapses, S., Heshka, S., and Heymsfield, S. B. (2007). MRI-measured Bone Marrow Adipose Tissue Is Inversely Related to DXA-Measured Bone mineral in Caucasian Women. Osteoporos. Int. 18 (5), 641-647. doi:10.1007/s00198-006-0285-9

Shen, W., Velasquez, G., Chen, J., Jin, Y., Heymsfield, S. B., Gallagher, D., et al. (2014). Comparison of the Relationship between Bone Marrow Adipose Tissue and Volumetric Bone mineral Density in Children and Adults. J. Clin. Densitom. 17 (1), 163-169. doi:10.1016/ j.jocd.2013.02.009

Suchacki, K. J., Tavares, A. A. S., Mattiucci, D., Scheller, E. L., Papanastasiou, G., Gray, C., et al. (2020). Bone Marrow Adipose Tissue Is a Unique Adipose Subtype with Distinct Roles in Glucose Homeostasis. Nat. Commun. 11 (1), 3097. doi:10.1038/s41467-020-16878-2

Suresh, S., de Castro, L. F., Dey, S., Robey, P. G., and Noguchi, C. T. (2019). Erythropoietin Modulates Bone Marrow Stromal Cell Differentiation. Bone Res. 7, 21. doi:10.1038/s41413-019-0060-0

Theill, L. E., Boyle, W. J., and Penninger, J. M. (2002). RANK-L and RANK: T Cells, Bone Loss, and Mammalian Evolution. Annu. Rev. Immunol. 20, 795-823. doi:10.1146/annurev.immunol.20.100301.064753

Tikhonova, A. N., Dolgalev, I., Hu, H., Sivaraj, K. K., Hoxha, E., CuestaDomínguez, Á., et al. (2019). The Bone Marrow Microenvironment at Single-Cell Resolution. Nature 569 (7755), 222-228. doi:10.1038/s41586019-1104-8

Tokoyoda, K., Zehentmeier, S., Hegazy, A. N., Albrecht, I., Grün, J. R., Löhning, M., et al. (2009). Professional Memory CD4+ T Lymphocytes Preferentially Reside and Rest in the Bone Marrow. Immunity 30 (5), 721-730. doi:10.1016/ j.immuni.2009.03.015

Ye, L., Fan, Z., Yu, B., Chang, J., Al Hezaimi, K., Zhou, X., et al. (2012). Histone Demethylases KDM4B and KDM6B Promotes Osteogenic Differentiation of Human MSCs. Cell Stem Cell 11 (1), 50-61. doi:10.1016/j.stem.2012.04.009

Yu, W., Zhong, L., Yao, L., Wei, Y., Gui, T., Li, Z., et al. (2021). Bone Marrow Adipogenic Lineage Precursors Promote Osteoclastogenesis in Bone Remodeling and Pathologic Bone Loss. J. Clin. Invest. 131 (2), e140214. doi:10.1172/jci140214

Yu, Y., Newman, H., Shen, L., Sharma, D., Hu, G., Mirando, A. J., et al. (2019). Glutamine Metabolism Regulates Proliferation and Lineage Allocation in Skeletal Stem Cells. Cel Metab. 29 (4), 966-978. e964. doi:10.1016/ j.cmet.2019.01.016

Zhong, L., Yao, L., Tower, R. J., Wei, Y., Miao, Z., Park, J., et al. (2020). Single Cell Transcriptomics Identifies a Unique Adipose Lineage Cell Population that Regulates Bone Marrow Environment. Elife 9, e54695. doi:10.7554/eLife.54695

Zhou, B. O., Yu, H., Yue, R., Zhao, Z., Rios, J. J., Naveiras, O., et al. (2017). Bone Marrow Adipocytes Promote the Regeneration of Stem Cells and Haematopoiesis by Secreting SCF. Nat. Cel Biol 19 (8), 891-903. doi:10.1038/ncb3570

Zhou, B. O., Yue, R., Murphy, M. M., Peyer, J. G., and Morrison, S. J. (2014). Leptin-receptor-expressing Mesenchymal Stromal Cells Represent the Main 
Source of Bone Formed by Adult Bone Marrow. Cell Stem Cell 15 (2), 154-168. doi:10.1016/j.stem.2014.06.008

Zinngrebe, J., Debatin, K.-M., and Fischer-Posovszky, P. (2020). Adipocytes in Hematopoiesis and Acute Leukemia: Friends, Enemies, or Innocent Bystanders? Leukemia 34 (9), 2305-2316. doi:10.1038/s41375-0200886-x

Zou, W., Rohatgi, N., Brestoff, J. R., Li, Y., Barve, R. A., Tycksen, E., et al. (2020). Ablation of Fat Cells in Adult Mice Induces Massive Bone Gain. Cel Metab. 32 (5), 801-813. e806. doi:10.1016/j.cmet.2020.09.011

Conflict of Interest: The authors declare that the research was conducted in the absence of any commercial or financial relationships that could be construed as a potential conflict of interest.
Publisher's Note: All claims expressed in this article are solely those of the authors and do not necessarily represent those of their affiliated organizations, or those of the publisher, the editors, and the reviewers. Any product that may be evaluated in this article, or claim that may be made by its manufacturer, is not guaranteed or endorsed by the publisher.

Copyright $\odot 2021$ Wang, Zhang, Wang, Chen and Su. This is an open-access article distributed under the terms of the Creative Commons Attribution License (CC BY).

The use, distribution or reproduction in other forums is permitted, provided the original author(s) and the copyright owner(s) are credited and that the original publication in this journal is cited, in accordance with accepted academic practice. No use, distribution or reproduction is permitted which does not comply with these terms. 